Imperial/TP/95-96/70

hep-th/9609212

\title{
'No force' condition and BPS combinations of p-branes in 11 and 10 dimensions
}

\author{
A.A. Tseytlin ${ }^{\star \dagger}$ \\ Theoretical Physics Group, Blackett Laboratory, \\ Imperial College, London SW7 2BZ, U.K.
}

\begin{abstract}
The condition of vanishing of static force on a q-brane probe in the gravitational background produced by another p-brane is used to give a simple derivation of the pair-wise intersection rules which govern the construction of BPS combinations of branes. These rules, while implied also by supersymmetry considerations, thus have purely bosonic origin. Imposing the no-force requirement makes possible to add branes 'one by one' to construct composite BPS configurations (with zero binding energy) of 2-branes and 5-branes in $\mathrm{D}=11$ and of various $\mathrm{p}$-branes in $\mathrm{D}=10$. The advantage of this elementary approach is its universality, i.e. the cases of different dimensions and different types of branes (e.g., NS-NS, R-R and 'mixed' combinations of NS-NS and R-R branes in D=10) are all treated in the same way.
\end{abstract}

September 1996

* e-mail address: tseytlin@ic.ac.uk

$\dagger$ Also at Lebedev Physics Institute, Moscow. 


\section{Introduction}

Recent developments in string theory suggest the importance of better understanding of the structure of composite p-brane solutions 10 and 11 dimensions. One is usually interested in stable extreme configurations which preserve some supersymmetry. For classical bosonic solutions the relevant condition can be stated as a special property of the corresponding background fields (e.g., special holonomy of appropriate connection), which, upon embedding of the solution in a supergravity theory, implies preservation of some amount of supersymmetry. It may be useful to try to distinguish the condition of residual supersymmetry from that of the BPS one since the latter concept is defined already in a bosonic theory 1 The BPS configurations with zero binding energy (the only ones which will be considered in this paper) are determined by solutions of linear Laplace equations and thus satisfy the 'no force' condition, allowing one to displace constituents at no cost in the energy.

A further clarification of the role of BPS condition in string theory may provide clues about M-theory. While in string theory there is a remarkable principle of the world-sheet conformal invariance that determines the higher-order corrections to the classical target space effective action, no similar principle is known in 11-dimensional theory. At the same time, several backgrounds for which the string $\sigma$-model has the property of exact conformal invariance (like the fundamental string and the solitonic 5-brane) are expressed in terms of functions which satisfy the Laplace equation and thus obey the BPS property. The Laplace equation condition directly follows from the special structure of background and the condition of conformal invariance (the vanishing of Laplacian effectively corresponds to marginality of relevant vertex operator). This suggests that some appropriate 'relaxation' of BPS condition may be a counterpart of conformal invariance of the string $\sigma$-model in the case of p-branes.

Below we shall demonstrate how one can determine the basic features of composite BPS p-brane backgrounds by considering a q-brane probe moving in a background produced by a p-brane source and findind which relative orientations of the probe and the source lead to 'no-force' condition. Having established which combination of a q-brane and a p-brane preserves the BPS property one may consider their composition as a source for a more complicated background. Next, one can add another m-brane probe, orient it in a 'no force' way, etc. This simple procedure works for M-branes of 11-dimensional theory as well as for various (NS-NS, R-R and mixed) p-brane configurations of $D=10$ type II theory. The results are consistent with other approaches in $D=10$ (D-brane supersymmetry analysis or study of potential between D-branes [1,2, 3, (4) $)$ and $D=11$ [5, 6, ], 8].

1 The BPS condition is obviously more general than that of residual supersymmetry: standard supersymmetry exists only in space-times of certain dimensions while classical bosonic BPS configurations are possible in any number of dimensions. 
The composite BPS solutions which will be considered in this paper are the ones with zero binding energy and the dimension of common transverse space $>2$ (so that they are localised in transverse space and have well-defined energy). In addition, there are two other classes of possible BPS configurations which will not be discussed. The first consists of 'no force' configurations with dimension of the transverse space being 1: (i) 'intersection' of two NS-NS 5-branes over a string in $D=10$ [9], S-dual intersection of two R-R 5-branes, and, more generally, D-brane configurations with the number of Dirichlet-Neumann directions equal to 8 [2].3, 团; (ii) 'intersection' of two 5-branes over a line in $D=11$ [8]. The second class includes $\frac{1}{2}$-supersymmetric solutions (sometimes obtained by applying S-duality in 10 or lower dimensions) which can be interpreted as strongly-coupled bound states with non-trivial binding energy: $1_{N S} \| 1_{R}$ [10], $0\|2,1\| 3$, etc. [2] in $D=10$, and $2 \| 5$ in $D=11$ [11,5.,12].

\section{2. $D=11$}

We shall first consider the $D=11$ case which is effectively simpler then 'dilatonic' $D=10$ one. Our starting point is the standard action for a p-brane moving in a background of D-dimensional metric $G_{\mu \nu}$ and $(p+1)$-form field $B_{\mu_{1} \ldots \mu_{p+1}}$

$$
\begin{gathered}
I_{p}=T_{p} \int d^{p+1} \sigma\left[\sqrt{-\operatorname{det} \hat{G}_{m n}}+\frac{1}{(p+1) !} \epsilon^{m_{1} \ldots m_{p+1}} \hat{B}_{m_{1} \ldots m_{p+1}}+\ldots\right] \\
\hat{G}_{m n}=G_{\mu \nu}(x) \partial_{m} x^{\mu} \partial_{n} x^{\nu}, \quad \hat{B}_{m_{1} \ldots m_{p+1}}=B_{\mu_{1} \ldots \mu_{p+1}}(x) \partial_{m_{1}} x^{\mu_{1}} \ldots \partial_{m_{p+1}} x^{\mu_{p+1}}
\end{gathered}
$$

It will be sufficient for our purposes to ignore other possible terms in the p-brane actions (e.g., terms involving world-volume vector or tensor fields). Adding this action as a source to $S=\frac{1}{2 \kappa^{2}} \int d^{D} x \sqrt{g}\left[R-\frac{1}{2(p+2) !} F_{p+2}^{2}\right], F_{p+2}=d B_{p+1}$ one finds the corresponding 'electric' p-brane solutions [13].

Let us choose the static gauge $x^{m}=\sigma^{m}(m=0,1, \ldots, p)$, so that

$$
\begin{gathered}
\hat{G}_{m n}=G_{m n}(x)+G_{i j}(x) \partial_{m} x^{i} \partial_{n} x^{j} \\
\hat{B}_{m_{1} \ldots m_{p+1}}=B_{m_{1} \ldots m_{p+1}}(x)+B_{m_{1} \ldots m_{p} i}(x) \partial_{m_{p+1}} x^{i}+\ldots
\end{gathered}
$$

For simplicity we assumed that the background fields do not depend on $x^{m}=y^{m}$, though this is not necessary. We have also assumed that $G_{m i}=0$, i.e. that the metric has a 'block-diagonal' structure. Expanding (2.1) in powers of derivatives of $x^{i}$ we get

$$
\begin{gathered}
I_{p}=T_{p} \int d^{p+1} \sigma\left[V(x)+\frac{1}{2} \gamma_{i j}^{k l} \partial_{k} x^{i} \partial_{l} x^{j}+\ldots\right], \\
V=\sqrt{-\operatorname{det} G_{m n}}+\frac{1}{(p+1) !} \epsilon^{m_{1} \ldots m_{p+1}} B_{m_{1} \ldots m_{p+1}}(x),
\end{gathered}
$$




$$
\gamma_{i j}^{k l}=\sqrt{-\operatorname{det} G_{m n}} G^{k l} G_{i j}
$$

where $V$ is the effective static potential. If $V$ is not constant there will be a force term in the corresponding equation of motion for the p-brane probe. Higher order terms in the expansion give 'velocity-dependent' corrections to the potential. The 'no-force' BPS configurations thus should lead to $V=0$ or $V=$ const. The resulting condition depends (i) on a type of p-brane probe, (ii) on a form of background fields, and (iii) on an orientation of the probe with respect to the background (i.e. to the source q-brane which produces it). Similar considerations were used in some special cases in [14, 15, 16, 13].

While the arguments below have a straightforward generalisation to the case of nondilatonic p-branes in $D$ dimensions, we shall consider explicitly the specific examples which are of most interest: 2-brane and 5-brane in 11 dimensions.

\subsection{2-brane probe in 2-brane background}

The extremal BPS background produced by a 2 -brane source is [15] $(i=1, . ., 8)$

$$
\begin{gathered}
d s_{11}^{2}=H^{1 / 3}(x)\left[H^{-1}(x)\left(-d t^{2}+d y_{1}^{2}+d y_{2}^{2}\right)+d x_{i} d x_{i}\right] \\
B_{3}=-H^{-1}(x) d t \wedge d y_{1} \wedge d y_{2}, \quad \partial^{2} H=0 .
\end{gathered}
$$

$H(x)$ is a harmonic function (which may depend only on part of $x$-coordinates, e.g., as a result of taking a periodic array of 1-center solutions).

Suppose we put a 2-brane probe in this background. If the probe is oriented parallel to the source 2-brane, i.e. it lies in the plane $y_{1}, y_{2}$, then the total effective potential $V$ vanishes because of the cancellation of the contributions of the 'Nambu' and 'Wess-Zumino' terms in $(2.5)$ 15

$$
V=\left[\left(H^{-2 / 3}\right)^{3}\right]^{1 / 2}-H^{-1}=0
$$

The cancellation obviously depends on the power $1 / 3$ in the prefactor in (2.7) (which is thus directly related to the dimension of the brane world-volume) and on the sign of the relative orientation of the source and the probe.

This implies that adding 2-branes parallel to the source 2-brane and considering the field they produce one should still get a BPS background. Indeed, the corresponding solution is given by (2.7),(2.8) with multicenter choice for $H$. The 'metric' $\gamma$ in (2.2) is flat in this case,

$$
\gamma_{i j}^{k l}=H^{-1}\left(H^{-2 / 3}\right)^{-1} H^{1 / 3} \eta^{k l} \delta_{i j}=\eta^{k l} \delta_{i j}
$$

2 Similar conclusion is reached if one does not fix the static gauge but just exands the action in powers of derivatives of $x^{i}: V=H^{-1} \sqrt{\left|\operatorname{det}\left(\partial_{m} y^{k} \partial_{n} y^{k}\right)\right|}-H^{-1} \operatorname{det}\left(\partial_{m} y^{k}\right)=0$. 
so that corrections to the force start at fourth order in derivatives. This is actually true in general for a p-brane source parallel to a BPS p-brane (for similar observations see, e.g., 13, 17, (4).

What happens if the probe is oriented orthogonally to the $y_{1}, y_{2}$ plane, e.g., if it lies in the $x_{1}, x_{2}$ plane, orthogonally intersecting the source 2-brane over one point? As follows from the structure of (2.7), 2.8), in this case the effective potential (2.5) receives contribution only from the first term ( $B_{3}$ has no components in $x^{i}$-directions). Counting the factors of $H$ coming from the common time direction and $x_{1}, x_{2}$ directions we find that they cancel out,

$$
V=\left[H^{-2 / 3}\left(H^{1 / 3}\right)^{2}\right]^{1 / 2}=1
$$

so that again there is no force on a static probe. In this case $\gamma_{i j}^{k l}$ in (2.2) is not constant,

$$
\gamma_{i^{\prime} j^{\prime}}^{k l}=G^{k l} G_{i^{\prime} j^{\prime}}=\gamma^{k l} \delta_{i^{\prime} j^{\prime}}, \quad \gamma^{00}=-H, \quad \gamma^{a b}=\delta^{a b}
$$

where $\left(i^{\prime}, j^{\prime}\right)$ correspond to the dimensions transverse to both source and the probe, and $(a, b)$ label two of $x^{i}$ dimensions along which the probe is lying. Thus here the corrections to the force start at second order in the expansion in power of velocity.

If, however, the 2-brane probe shares one spatial dimension with the 2-brane source, the potential is

$$
V=\left[\left(H^{-2 / 3}\right)^{2} H^{1 / 3}\right]^{1 / 2}=H^{-1 / 2} \neq \text { const },
$$

i.e. the configuration of the two 2-branes orthogonally intersecting over a line will not be in equilibrium.

The conclusion is that the source composed of the two 2-branes orthogonally intersecting over a point should produce a static BPS background. Indeed, the corresponding extremal field configuration exists and is an obvious generalisation of (2.7), (2.8) [5, 6] $(i=1, . ., 6)$

$$
\begin{gathered}
d s_{11}^{2}=\left(H_{1} H_{2}\right)^{1 / 3}(x)\left[-\left(H_{1} H_{2}\right)^{-1}(x) d t^{2}+H_{1}^{-1}(x)\left(d y_{1}^{2}+d y_{2}^{2}\right)\right. \\
\left.+H_{2}^{-1}(x)\left(d y_{3}^{2}+d y_{4}^{2}\right)+d x_{i} d x_{i}\right], \\
B_{3}=-H_{1}^{-1}(x) d t \wedge d y_{1} \wedge d y_{2}-H_{2}^{-1}(x) d t \wedge d y_{3} \wedge d y_{4}, \quad \partial^{2} H_{1,2}=0 .
\end{gathered}
$$

This $2 \perp 2$ solution corresponds to 2 -brane sources being 'delocalised' in the internal dimensions of each other (equivalently, it can be interpreted as an anisotropic 4-brane [18]). The structure of the background is precisely such that adding a 2-brane probe parallel to each of the two 2-brane planes we get zero force, in agreement with the possibility of multicenter choices for each of the two harmonic functions $H_{1,2}$ [6]. The metric $\gamma$ in this case is not constant (if the probe is parallel to the first 2-brane then $\gamma^{00}=-H_{2}$, in agreement 
with (2.12)). This property - the presence of velocity squared corrections to the force - is generic to backgrounds produced by BPS superpositions of two or more p-branes.

If the 2-brane probe is oriented orthogonally to each of the two 2-brane sources we get again $V=1$ as in (2.11). Hence there exists the $2 \perp 2 \perp 2$ solution where all of the 2-branes intersect only over one point [5, 6, 7, 8], etc. This provides a simple explanation of the 'harmonic function rule' of [6], according to which each square of differential of a coordinate of a BPS composition of branes should be multiplied by the inverse power of the product of harmonic functions of branes it belongs to (relative to the common transverse space interval).

The form of intersecting solutions is thus not accidental and, not unexpectedly, is intimately connected with the structure of the corresponding p-brane actions.

\subsection{Intersections of 5-branes with 5-branes and 2-branes}

The story in the 5 -brane case is very similar. The basic 'magnetic' solution in $D=11$ is 18

$$
\begin{gathered}
d s_{11}^{2}=H^{2 / 3}(x)\left[H^{-1}(x)\left(-d t^{2}+d y_{1}^{2}+\ldots+d y_{5}^{2}\right)+d x_{i} d x_{i}\right], \\
F_{4}=d B_{3}=* d H, \quad \partial^{2} H=0,
\end{gathered}
$$

where $i=1, . ., 5$ and $* d H$ is the dual form in $R_{x}^{5}$. The 5-brane probe couples to the dual 'electric' potential $B_{6}$ defined by $d B_{6}=* d B_{3}$, where the dual is taken with respect to the full 11-dimensional metric (corrections due to the presence of the Chern-Simons $d B_{3} \wedge B_{3} \wedge B_{3}$ term in $D=11$ action vanish for this solution) 3

$$
B_{6}=-H^{-1}(x) d t \wedge d y_{1} \wedge d y_{2} \wedge d y_{3} \wedge d y_{4} \wedge d y_{5}
$$

The background (2.16), 2.18) describes the 'electric' 5-brane solution of the 'Einstein gravity +6 -form' action.

If the 5 -brane probe is parallel to the 5-brane plane $\left(y_{1}, \ldots, y_{5}\right)$ the static potential (2.5) vanishes due to the cancellation of the two terms in (2.5) as in (2.9),

$$
V=\left[\left(H^{-1 / 3}\right)^{6}\right]^{1 / 2}-H^{-1}=0
$$

implying the existence of the BPS configuration of multiple parallel 5-branes (which is described by the multicenter version of (2.16), (2.17)).

If the 5-brane probe is oriented so that it orthogonally intersects the 5-brane source over $n<5$ of $y_{m}$ dimensions, then, as follows from (2.16), (2.3), (2.5),

$$
V=\left[\left(H^{-1 / 3}\right)^{n+1}\left(H^{2 / 3}\right)^{5-n}\right] 1 / 2=H^{(3-n) / 2} .
$$

3 Other details of the structure of $D=115$-brane probe action (see [19] and refs. there) will not be relevant below. 
Thus the no-force condition is realised only if the two 5-branes intersect over a 3-space, in agreement with the suggestion in [5]. The $5 \perp 5$ background produced by two 5 -branes orthogonally intersecting over a 3-space (and delocalised in the common internal 7-space) is given by [5, 6 ]

$$
\begin{gathered}
d s_{11}^{2}=\left(H_{1} H_{2}\right)^{2 / 3}(x)\left[\left(H_{1} H_{2}\right)^{-1}(x)\left(-d t^{2}+d y_{1}^{2}+d y_{2}^{2}+d y_{3}^{2}\right)\right. \\
\left.+H_{1}^{-1}(x)\left(d y_{4}^{2}+d y_{5}^{2}\right)+H_{2}^{-1}(x)\left(d y_{6}^{2}+d y_{7}^{2}\right)+d x_{i} d x_{i}\right], \\
F_{4}=* d H_{1}(x) \wedge d y_{4} \wedge d y_{5}+* d H_{2}(x) \wedge d y_{6} \wedge d y_{7}, \quad \partial^{2} H=0,
\end{gathered}
$$

where $* d H$ is the dual with respect to the transverse space $R_{x}^{3}$, so that

$$
B_{6}=-H_{1}^{-1}(x) d t \wedge d y_{1} \wedge d y_{2} \wedge d y_{3} \wedge d y_{4} \wedge d y_{5}-H_{2}^{-1}(x) d t \wedge d y_{1} \wedge d y_{2} \wedge d y_{3} \wedge d y_{6} \wedge d y_{7}
$$

This can be interpreted as a background produced by a source built out of two 'elementary' 5 -branes oriented along $\left(y_{1}, y_{2}, y_{3}, y_{4}, y_{5}\right)$ and $\left(y_{1}, y_{2}, y_{3}, y_{6}, y_{7}\right)$, i.e. having a common 3 space.

Putting a 5 -brane source in this $5 \perp 5$ background one learns that the no-force condition is satisfied only if the probe is parallel to any of the two constituent 5-branes or if it intersects any of the two over a 3-space. That, in turn, implies the existence of the BPS configuration of the three intersecting 5-branes, each pair sharing a 3 -space (and thus all three having one common 'string' direction) [5,6]. One may continue the process of adding new 5-branes, getting new BPS configurations as long as the above intersection rule is satisfied and one does not exceed the total number 10 of spatial dimensions [8].

The next natural step is to consider a 2-brane probe in the 5-brane background (2.16), (2.22). One learns that the potential (2.5) is constant only if the 2-brane shares one common dimension with the 5 -brane, i.e. it intersects the $y_{1}, \ldots, y_{5}$ hyperplane over a line and has the second direction oriented along one of the transverse coordinates $x^{i}$. In this case $V=\left[\left(H^{-1 / 3}\right)^{2} H^{2 / 3}\right]^{1 / 2}=1$ (the WZ term does not contribute here). Not surprisingly, the same conclusion is reached by studying the 5-brane probe in the background produced by the 2-brane source (2.7),(2.8). As far as the potential is concerned, the picture is completely symmetric with respect to the source and the probe: what we are discussing is just the static force between the two branes interacting via massless graviton and 3 -form field exchanges (described by $R+F_{4}^{2}$ action).

As a result, there should exist 'mixed' BPS backgrounds produced by intersecting 2-branes and 5-branes under the rule that any 2-brane and 5-brane can intersect over a line, 2 and 2 can intersect over a point, and 5 and 5 can intersect over a 3 -space, namely, $2 \perp 5,2 \perp 2 \perp 5,2 \perp 5 \perp 5,2 \perp 2 \perp 5 \perp 5$, etc. [6, [7, 8]. This conclusion is true in the bosonic sector of the theory and, as clear from the above, is not directly related to the embedding in the supergravity theory (residual supersymmetry is only a particular consequence of the special geometrical properties of the bosonic BPS backgrounds). 


\section{3. $\mathrm{D}=10$}

Let us now demonstrate how similar simple considerations imply the rules of constructing composite BPS configurations of various branes in 10-dimensional type IIA,B theories.

\section{1. $p$-branes of NS-NS sector}

The NS-NS fundamental string background is encoded in the following string $\sigma$-model Lagrangian (written in the conformal gauge, and omitting the coupling to the dilaton $\phi$ ) [14, 20,

$$
L=H^{-1}(x) \partial_{+} u \partial_{-} v+\partial_{+} x_{i} \partial_{-} x_{i}, \quad \partial^{2} H=0,
$$

where $u, v=y \mp t$, i.e.

$$
d s_{10}^{2}=H^{-1}(x)\left(-d t^{2}+d y\right)^{2}+d x_{i} d x_{i}, \quad B_{2}=-H^{-1} d t \wedge d y, \quad e^{2 \phi}=H^{-1} .
$$

The action and potential for a classical test string moving in a $D=10$ type II theory background is given by $(2.1),(2.4),(2.5)$. If the probe is oriented parallel to $y$ we get zero potential $V$ (2.5) and flat metric $\gamma$ (2.6) [13] (as can be seen also by evaluating $L$ (3.1) in the static gauge $\left.t=\tau, y=\sigma, \partial_{+} u=0, \partial_{-} v=0\right)$. This is related, via dimensional reduction, to analogous facts about $D=112$-branes [15]. If the string probe is oriented orthogonally to the test string the potential is non-constant $\left(V=H^{-1 / 2}\right)$, i.e. this is not a BPS configuration (cf. the presence of a non-zero force between the $D=112$-branes which have one common direction).

The NS-NS solitonic 5-brane background is described by 21,22

$$
d s_{10}^{2}=-d t^{2}+d y_{1}^{2}+\ldots+d y_{5}^{2}+H(x) d x_{i} d x_{i}, \quad d B_{2}=* d H, \quad e^{2 \phi}=H
$$

There is obviously no force $(V=1)$ on the string probe parallel to the 5 -brane directions $y_{m}$, implying the existence of the composite 1\|5 BPS configuration [23] (which can be viewed also as a reduction of $2 \perp 5$ in $D=11$ [6]). The 'metric' $\gamma(2.6$ ) is non-trivial $\left(\gamma_{i j}^{k l}=H(x) \eta^{k l} \delta_{i j}\right)$, i.e. there are velocity squared corrections to the force. Explicitly, the static-gauge action of a string probe moving in a single 5-brane background is ( $s=$ $2,3,4,5 ; i=1,2,3,4)$

$$
I_{1}=T_{1} \int d^{2} \sigma\left[1+\frac{1}{2} \partial_{n} x^{s} \partial^{n} x^{s}+\frac{1}{2} H(x) \partial_{n} x^{i} \partial^{n} x^{i}+\ldots\right], \quad H=1+\frac{Q_{5}}{x_{i}^{2}}
$$

The same action is found for a string probe moving in the 1\|5 bound state background [23] $\left(s_{10}^{2}=H_{1}^{-1}\left(-d t^{2}+d y_{1}^{2}\right)+d y_{s} d y_{s}+H_{5} d x_{i} d x_{i}\right.$, etc. $)$ parallel to the source string: all dependence on $H_{1}$ cancels out. 
If the string probe is oriented orthogonally to the 5-brane (i.e. the string and 5brane intersect at one point) then $V=H^{1 / 2} \neq$ const, i.e. there is no stable $1_{N S} \perp 5_{N S}$ configuration.

The action for a NS-NS 5-brane probe moving in $D=10$ background [24]

$$
I_{5_{N S}}=T_{5} \int d^{6} \sigma\left[e^{-2 \phi} \sqrt{-\operatorname{det} \hat{G}_{m n}}+\frac{1}{6 !} \epsilon^{m_{1} \ldots m_{6}} \hat{B}_{m_{1} \ldots m_{6}}+\ldots\right], \quad d B_{6}=* d B_{2},
$$

differs from (2.1) by an extra dilaton factor in the first term. also in the corresponding potential $V$ and metric $\gamma$ (cf. (2.5),(2.6)).

The potential for a 5-brane probe moving in the fundamental string background and oriented parallel to the string source is thus

$$
V=e^{-2 \phi} \sqrt{-\operatorname{det} G_{m n}}=H\left[\left(H^{-1}\right)^{2}\right]^{1 / 2}=1
$$

This is of course the same conclusion as reached above for a string probe moving in the 5-brane background.

If we consider a 5-brane probe in a 5-brane background then the potential vanishes if the probe is parallel to the source (the contribution of the WZ term is then non-zero). If the source and the probe share $n<5$ spatial dimensions then

$$
V=e^{-2 \phi} \sqrt{-\operatorname{det} G_{m n}}=H^{-1}\left(H^{5-n}\right)^{1 / 2}=H^{(3-n) / 2}
$$

i.e. the static $D=10$ NS-NS 5-branes are allowed to intersect over 3 -spaces without experiencing a force, just like the 5-branes of 11-dimensional theory (cf. (2.20)). This is consistent with the fact that the $5 \perp 5$ background in $D=11$ gives the $5_{N S} \perp 5_{N S}$ solution in $D=10$ upon direct (or 'periodic array') dimensional reduction along one of the dimensions of the common transverse 3 -space. The metric $\gamma$ is non-trivial in all cases except $5_{N S} \| 5_{N S}$.

Having established the rules of no-force pair-wise combinations of NS-NS strings and 5 -branes one is able to determine possible composite BPS configurations, e.g., $1_{N S} \| 5_{N S} \perp 5_{N S}$, etc.

4 This factor appears upon dimensional reduction if one starts with the $D=11$-brane action (2.1) and uses the ansatz (relating the $D=11$ and $D=10$ space-time actions) $d s_{11}^{2}=$ $e^{\frac{4}{3} \phi} d x_{11}^{2}+e^{-\frac{2}{3} \phi} d s_{10}^{2}$, where $d s_{10}^{2}$ corresponds to the string-frame metric. 


\section{2. $R$ - $R$ branes}

The p-branes supported by a $\mathrm{R}$ - $\mathrm{R}$ charge (Dp-branes) are described by the actions similar to (2.1) (we shall assume that $p<7$ )

$$
I_{p_{R R}}=T_{p} \int d^{p+1} \sigma\left[e^{-\phi} \sqrt{-\operatorname{det}\left(\hat{G}_{m n}+\hat{B}_{m n}\right)}+\frac{1}{(p+1) !} \epsilon^{m_{1} \ldots m_{p+1}} \hat{C}_{m_{1} \ldots m_{p+1}}+\ldots\right]
$$

with the extra dilaton factor, the $\hat{B}_{m n}=B_{\mu \nu}(x) \partial_{m} x^{\mu} \partial_{n} x^{\nu}$ term in the 'Nambu' part and the $\mathrm{R}-\mathrm{R}(p+1)$-form field in the WZ part (dots stand for other terms involving worldvolume vector field [25,26]). $B_{\mu \nu}$ effectively couples only to the 'transverse' coordinates, so that the analogs of the potential $V$ and the metric $\gamma$ in $(2.5),(2.6)$ are

$$
\begin{gathered}
V=e^{-\phi} \sqrt{-\operatorname{det} G_{m n}}+\frac{1}{(p+1) !} \epsilon^{m_{1} \ldots m_{p+1}} C_{m_{1} \ldots m_{p+1}}(x), \\
\gamma_{i j}^{k l}=V G^{k l}\left(G_{i j}+B_{i j}\right) .
\end{gathered}
$$

The extremal type II background produced by a R-R charge (or a Dp-brane source) 27] can be represented as

$$
d s_{10}^{2}=H^{1 / 2}\left[H^{-1}\left(-d t^{2}+d y_{1}^{2}+\ldots+d y_{p}^{2}\right)+d x_{i} d x_{i}\right], \quad e^{2 \phi}=H^{(3-p) / 2}
$$

with $C_{p+1}=-H^{-1} d t \wedge d y_{1} \wedge \ldots \wedge d y_{p}$ in the 'electric' cases and $d C_{7-p}=* d H$ in the 'magnetic' ones.5

Consider a Dq-brane probe moving in a Dp-brane background. If $p=q$ and the probe and the source are parallel to each other, then $V=H^{-1}-H^{-1}=0, \gamma_{i j}^{k l}=\eta^{k l} \delta_{i j}$ (in agreement with similar conclusions in [17,4]). If the probe and the source share $n$ common spatial dimensions then the resulting potential $V(3.9)$ and the 00-component of the metric $\gamma$ (3.9) are given by (the WZ term does not contribute in this case)

$$
\begin{gathered}
V=H^{-(3-p) / 4}\left[\left(H^{-1 / 2}\right)^{1+n}\left(H^{1 / 2}\right)^{q-n}\right]^{1 / 2}=H^{(p+q-2 n-4) / 4} \\
\gamma_{i j}^{00}=H^{(p+q-2 n-4) / 4} H^{1 / 2} H^{1 / 2} \delta_{i j}=H^{(p+q-2 n) / 4} \delta_{i j}
\end{gathered}
$$

The 'no-force' condition

$$
p+q-2 n=4
$$

is, as expected, symmetric under interchanging of the probe and the source. It is consistent with the condition of unbroken $\frac{1}{4}$ supersymmetry, or the vanishing of static potential (1loop vacuum energy of the open superstring in the presence of D-branes) derived using

5 A simple 'explanation' of the structure of the metric in (3.10) can be given by using formal T-duality considerations starting from flat space (or 9-brane) [28]. For $p=1,5$ this structure is implied also by the type IIB $S L(2)$-duality relation to the NS-NS string and 5-brane backgrounds. 
the open string theory representation of D-branes [2, 3, 4], namely, that the number of Dirichlet-Neumann directions $(=p+q-2 n)$ should be 4 (or 8), as well as with T-duality considerations 28,29 .6

The allowed configurations are thus

$$
\begin{gathered}
n=0: 4\left\|0,3 \perp 1_{R}, 2 \perp 2 ; \quad n=1: 5_{R}\right\| 1_{R}, 4 \perp 2,3 \perp 3 ; \\
n=2: 6\left\|2,5_{R} \perp 3,4 \perp 4 ; \quad n=3: 7\right\| 3,6 \perp 4,5_{R} \perp 5_{R},
\end{gathered}
$$

with the metric $\gamma$ being non-trivial in all cases except $p=q=n$, in agreement with the presence of velocity-squared terms in the force as computed in [17,4. For example, the action for a 0-brane probe moving in a 4-brane background is thus (cf. (3.4))

$$
I_{0}=T_{0} \int d \tau\left[1-\frac{1}{2} \partial_{\tau} x^{s} \partial_{\tau} x^{s}-\frac{1}{2} H(x) \partial_{\tau} x^{i} \partial_{\tau} x^{i}+\ldots\right], \quad H=1+\frac{Q_{4}}{x_{i}^{3}}
$$

where $x^{s}$ are 4 directions parallel to the 4 -brane and $x^{i}$ are 5 transverse directions. This reproduces the moduli space metric of the D0-D4 system discussed in [30]. As might be expected, the same action is found for a 0-brane moving in the $4 \| 0$ bound state background [6] $\left(d s_{10}^{2}=\left(H_{0} H_{4}\right)^{1 / 2}\left[-\left(H_{0} H_{4}\right)^{-1} d t^{2}+H_{4}^{-1} d y_{s} d y_{s}+d x_{i} d x_{i}\right], e^{2 \phi}=H_{0}^{3 / 2} H_{4}^{-1 / 2}\right.$, etc. $)$, i.e. the dependence on $H_{0}$ cancels out. The action for a D-string in the D5-brane background is, of course, equivalent to (3.4).

'Adding' Dp-branes one by one according to these pair-wise superposition rules one can explicitly construct the backgrounds produced by several intersecting D-branes as sources, e.g., $2 \perp 2 \perp 2,1 \perp 3 \perp 3,3 \perp 3 \perp 3 \perp 3$, etc. [6, [7,31]. By considering a test D-brane propagating in these composite backgrounds one finds that the no-force condition is satisfied if the probe is oriented with respect to any of the constituent branes according to (3.13),(3.14).

\section{3. 'Mixed' configurations of NS-NS and R-R branes}

Let us now study the Dp-brane probes moving in the NS-NS string (3.2) and 5brane (3.3) backgrounds, and vice versa, the elementary string and NS-NS 5-brane probes moving in the R-R p-brane background (3.10). The resulting no-force conditions should of course be invariant under interchange of the probe and the source. This provides a useful consistency check.

6 The case of $p+q-2 n=8$ (which includes, e.g., $n=0: 5 \perp 3,4 \perp 4 ; n=1: 5 \perp 5$ ) is special as it corresponds to solutions with 1-dimensional common transverse space. The correspondence between open string representation of D-branes and classical backgrounds is not clear in this case, as the solutions (like the ones in [9,8]) are 'localised' and have too few isometries, while D-branes in the open string picture are all simply related by T-duality transformations. 
If the Dp-brane probe is placed in the fundamental string background so that the string source is orthogonal to the Dp-brane hyperplane then the resulting potential (3.9) is constant due to the cancellation between the dilaton factor and the factor of the time component of the metric in (3.2), $V=H^{1 / 2} H^{-1 / 2}=1$. Thus there should exist stable configurations where a fundamental string orthogonally intersects a D-brane over a point, i.e. $1 \| 0,1_{N S} \perp 1_{R}, 1_{N S} \perp 3,1_{N S} \perp 5_{R}$, etc.

If the Dp-brane with $p>0$ is parallel to the NS-NS string source then $V$ (3.9) is nontrivial

$$
V=H^{1 / 2}\left(H^{-2}\right)^{1 / 2}=H^{-1 / 2},
$$

i.e. ' $1_{N S} \|$ Dp-brane' configurations do not satisfy the 'no-force' condition. Note in this connection that the BPS bound state $1_{N S} \| 1_{R}$ [10] has a non-vanishing binding energy (similar bound states are $2 \| 5$ in $D=11$ [11] and $2\|0,3\| 1,4 \| 2$, etc. in $D=10$ [2, 33, 12]). Such configurations (which are $\frac{1}{2}$-supersymmetric) have a different ('strong-coupling') nature than the BPS bound states with zero binding energy discussed in this paper (which preserve $\frac{1}{4}$ or less of maximal supersymmetry).

For a Dp-brane probe placed in the solitonic 5-brane background (3.3) so that the probe and the source are parallel to $n$ common spatial dimensions (with $p-n \leq 4$ to fit into the $D=10$ space-time) we find that $V$ in (3.9) is given by

$$
V=H^{-1 / 2}\left(H^{p-n}\right)^{1 / 2}=H^{(p-n-1) / 2} .
$$

The stable configurations must have $p-n=1$, i.e. all but one dimension of the $D$-brane should be parallel to the NS-NS 5-brane, i.e.

$$
\begin{aligned}
& n=0: 5_{N S} \perp 1_{R} ; \quad n=1: 5_{N S} \perp 2 ; \quad n=2: 5_{N S} \perp 3 ; \\
& n=3: 5_{N S} \perp 4 ; \quad n=4: 5_{N S} \perp 5_{R} ; \quad n=5: 5_{N S} \| 6 .
\end{aligned}
$$

These results are consistent with some of the already obtained above: $5_{N S} \perp 1_{R}$ is related to $5_{R} \perp 1_{N S}$ and $5_{N S} \perp 3$ to $5_{R} \perp 3$ by S-duality of type IIB theory, $5_{N S} \perp 2$ of type IIA theory is the direct dimensional reduction of $5 \perp 2$ in 11 dimensions, $5_{N S} \perp 4$ is the reduction of 11-dimensional $5 \perp 5$.

Equivalent conclusions are, indeed, reached by considering the NS-NS string or 5brane probes moving in the Dp-brane background (3.10). The test string 'feels' only the metric in (3.10) while the test 5-brane is directly coupled only to the metric and the dilaton components of the Dp-brane background. If the string is oriented orthogonally to the Dp-brane, then $V=\left[H^{-1 / 2} H^{1 / 2}\right]^{1 / 2}=1$, while if it is parallel to the Dp-brane then $V=\left[\left(H^{-1 / 2}\right)^{2}\right]^{1 / 2}=H^{-1 / 2}$, in agreement with (3.16). A test NS-NS 5-brane parallel to $n$ spatial dimensions of the Dp-brane has the potential (3.7)

$$
V=H^{-(3-p) / 2}\left[\left(H^{-1 / 2}\right)^{n+1}\left(H^{1 / 2}\right)^{5-n}\right]^{1 / 2}=H^{(p-n-1) / 2},
$$

i.e. the same as in (3.17).

7 This is an analogue, for the static classical BPS backgrounds, of the expectation that the fundamental strings can 'end' on D-branes [32,2]. 


\section{Concluding remarks}

The aim of the present paper was to demonstrate how one can determine the structure of composite BPS configurations of p-branes using as an input only the knowledge of (i) the background fields produced by a single brane source and (ii) the basic terms in the action for a p-brane probe. The form of the background encodes the information about relevant massless fields and their couplings to the brane.

The resulting rules of superposing branes in $D=10$ are, essentially, implied by the rules in $D=11$. The explicitly known BPS configurations in $D=11$ ('boosted' 2-brane $2 \uparrow$, 'boosted' 5 -brane $5 \uparrow$, intersections $2 \perp 2, \ldots, 2 \perp 5 \perp 5$, etc., intersections with 'boosts' $2 \perp 5 \uparrow$, etc. [6, ]1) may be viewed as an economic way of describing, via dimensional reduction, various 'mixed' backgrounds in $D=10$. If the $D=11$ configuration satisfies the 'no-force' rules, the same will be true for $D=10$ configurations which are related to it by dimensional reduction.

To illustrate this, let us consider some examples, following [6,7]. $2 \uparrow$ leads to two IIA solutions in $D=10$ : (i) reducing along the boost direction we get $1_{N S} \| 0$, i.e. a bound state of a fundamental string and a 0-brane ( $\mathrm{T}$-dual to the boosted R-R string of type IIB, $1_{R R} \uparrow$ ); (ii) reducing along the other internal direction gives $1_{N S} \uparrow$ (T-duality gives back the boosted NS-NS string, now as a IIB solution). Note that the two different reductions of $D=11$ solution are related by T- and S- dualities in $D=10$. Similar relations are often true in other cases. For $5 \uparrow$ one finds: (i) reducing along the boost direction we get $4 \| 0$, i.e. a bound state of a 4 -brane and a 0 -brane (T-dual to $3 \perp 1_{R}$ type IIB solution); (ii) reducing along any other internal direction gives $4 \uparrow$; T-duality then gives either $(3 \uparrow)_{1}$ (the subscript indicates the number of extra compact isometric transverse dimensions, the total number of which remains the same in the process of reduction and T-duality) or $3 \perp 1_{N S} .2 \perp 2$ in $D=11$ reduces to $2 \perp 1$.

$2 \perp 5$ has the following reductions to $D=10$ : (i) $2 \perp 4$ (T-dual to $1_{R} \| 5_{R}, 3 \perp 3,\left(1_{R} \perp 3\right)_{1}$ ); (ii) $1 \perp 4$ (T-dual to $5_{R} \uparrow,\left(1_{N S} \perp 3\right)_{1}$ ); (iii) $1 \| 5$ (T-dual to $5_{N S} \uparrow$ and $1_{N S} \| 5_{N S}$ as a IIB solution, related by S-duality to $\left.1_{R} \| 5_{R}\right) .5 \perp 5$ reduces to: (i) $5 \perp 4$ (T-dual to $\left(5_{N S} \| 3\right)_{1}$ and $\left.5_{N S} \perp 3\right)$; (ii) $4 \perp 4$ (T-dual to $(3 \perp 3)_{1}$ and $\left.3 \perp 5_{R}\right)$.

Similar analysis can be repeated for $2 \perp 2 \perp 2,2 \perp 2 \perp 5$, etc. As a result, starting with one configuration in $D=11$ and reducing it in different ways one gets various BPS configurations in $D=10$ (with some being related by T-duality and type IIB S-duality), all of which, remarkably, having a common origin in $D=11$ theory.

8 The knowledge of explicit solution for a 0-brane lying on a closed string $(1 \| 0)$ is interesting since this is the only case where an 'intersecting' configuration formally looks like an 'ending' configuration (or like an open string wound around the compact direction, with both ends attached to the 0-brane). Note, however, that starting with $2 \uparrow$ in $D=11$ we are getting a 'smeared' solution, i.e. the 0 -brane is not localised on the fundamental string (the harmonic functions for the string and the 0-brane both depend only on 8 transverse directions). 


\section{Acknowledgements}

I am grateful to I.R. Klebanov for useful discussions of related issues. This work was partially supported by PPARC, the European Commission TMR programme ERBFMRXCT96-0045 and NATO grant CRG 940870. 


\section{References}

[1] J. Polchinski, Phys. Rev. Lett. 75 (1995) 4724, hep-th/9510017.

[2] J. Polchinski, S. Chaudhuri and C.V. Johnson, hep-th/9602052.

[3] M.B. Green and M. Gutperle, hep-th/9604091.

[4] G. Lifschytz, hep-th/9604156.

[5] G. Papadopoulos and P.K. Townsend, hep-th/9603087.

[6] A.A. Tseytlin, Nucl. Phys. B475 (1996) 149, hep-th/9604035.

[7] I.R. Klebanov and A.A. Tseytlin, Nucl. Phys. B475 (1996) 179, hep-th/9604166.

[8] J.P. Gauntlett, D.A. Kastor and J. Traschen, hep-th/9604179.

[9] R. Khuri, Phys. Rev. D48 (1993) 2947.

[10] J.H. Schwarz, Phys. Lett. B367 (1996) 97, hep-th/9510086, hep-th/9601077.

[11] J.M. Izquierdo, N.D. Lambert, G. Papadopoulos and P.K. Townsend, Nucl. Phys. B460 (1996) 560, hep-th/9508177.

[12] G. Papadopoulos and P.K. Townsend, hep-th/9609095.

[13] M.J. Duff, R. Khuri and J.X. Lu, Phys. Rept. 259 (1995) 213, hep-th/9412184.

[14] A. Dabholkar, G.W. Gibbons, J. Harvey and F. Ruiz Ruiz, Nucl. Phys. B340 (1990) 33; A. Dabholkar and J. Harvey, Phys. Rev. Lett. 63 (1989) 478.

[15] M.J. Duff and K.S. Stelle, Phys. Lett. B253 (1991) 113.

[16] C.G. Callan and R.R. Khuri, Phys. Lett. B261 (1991) 363.

[17] C. Bachas, Phys. Lett. B374 (1996) 37, hep-th/9511045.

[18] R. Güven, Phys. Lett. B276 (1992) 49.

[19] E. Bergshoeff, M. de Roo and T. Ortín, hep-th/9606118.

[20] G.T. Horowitz and A.A. Tseytlin, Phys. Rev. D51 (1995) 2896, hep-th/9409021.

[21] M.J. Duff and J.X. Lu, Nucl. Phys. B354 (1991) 141.

[22] C.G. Callan, J.A. Harvey and A. Strominger, Nucl. Phys. B359 (1991) 611.

[23] A.A. Tseytlin, Mod. Phys. Lett. A11 (1996) 689, hep-th/9601177.

[24] C.G. Callan, J.A. Harvey and A. Strominger, Nucl. Phys. B367 (1991) 60.

[25] R.G. Leigh, Mod. Phys. Lett. A4 (1989) 2767.

[26] M. Douglas, hep-th/9512077.

[27] G.T. Horowitz and A. Strominger, Nucl. Phys. B360 (1991) 197.

[28] K. Behrndt, E. Bergshoeff and B. Janssen, hep-th/9604168.

[29] E. Bergshoeff and M. de Roo, hep-th/9603123.

[30] M.R. Douglas, D. Kabat, P. Pouliot and S.H. Shenker, hep-th/9608024.

[31] V. Balasubramanian and F. Larsen, hep-th/9604189.

[32] A. Strominger, Phys. Lett. B383 (1996) 44, hep-th/9512059.

[33] G. Papadopoulos, hep-th/9604068. 